\title{
Virtual Space Computer Games with a Floor Sensor Control Human Centred Approach in the Design Process
}

\author{
Jaana Leikas, Antti Väätänen, \\ Veli-Pekka Räty \\ VTT Information Technology \\ P.O.Box 1206 \\ FIN-33101 Tampere, FINLAND \\ +35833163111 \\ jaana.leikas@vtt.fi, antti.vaatanen@vtt.fi,veli-pekka.raty@vtt.fi
}

\begin{abstract}
Traditionally computer games are played with a keyboard and a mouse or a joystick. The playing relies mainly on visual and auditory senses. Tactile or haptic user interfaces and natural movements of the human being, e.g. running, are seldom utilised in computer games. The Lumetila project (Virtual Space - User Interfaces of the Future) aims at developing a "natural" user interface in a computer game where the user uses his body movements to control the game. To create an immersive, captivating and highly usable game, the development will be carried out in the context and practice of Human-Centred Design approach, where the computer game is designed and evaluated with end-users in every step of the iterative design process.
\end{abstract}

\section{Keywords}

Computer game, floor sensor, virtual space, user interface, human-centred design, usability

\section{INTRODUCTION}

Traditionally, computer games are played alone, the player against the computer, or in a two player game mode. The narration and the plot of the computer games have changed only little during the last decades, although effective computers and high resolution displays have added entertainment value to the games. Generally, the game user interface still contains a computer with a display and a keyboard, as well as a mouse or a joystick as a game controller. At the moment, the development of computer game controllers is rapid producing advanced ways for controlling computer games on the market. Force feedback joysticks and steering wheels are becoming controllers in computer games. However, these solutions still rely on the old way of controlling the game by using mainly one's hands and fingers.

Although technology enables already more accurate and more versatile sensors for recognising body movements of the players, only few computer games to make use of this technology have been developed.
The Lumetila project aims at exploring how people can, and how willing they are, to use their own body to control a computer game, and how they can interact with the computer game and with other players by moving around in a room. Our approach is to weaken the boundaries between the room surrounding the players and the interactive virtual space. With effects equipment and computer software we will create new immersive virtual spaces (Figure 1).

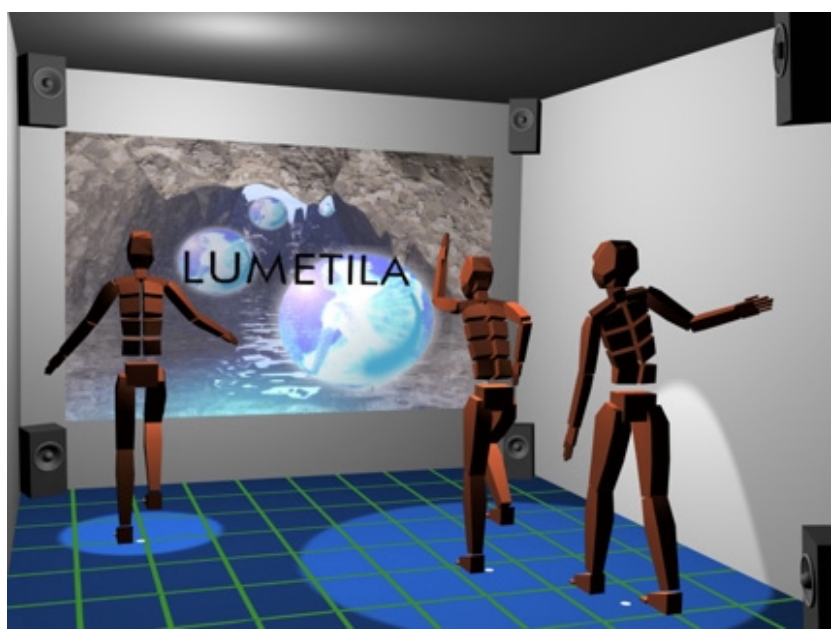

Figure 1. Visualisation of the Lumetila - Virtual Space (image by Tiina Kymäläinen, VTT Information Technology)

\section{THE DESIGN PROCESS}

There are several methodologies and different methods to carry out the design work. We chose the Human-Centred Design (HCD) approach to the Lumetila design. This approach aims at utilising the opinions of the end-users of the product as efficiently as possible in different stages of the development process. According to the approach, people who develop new applications have continuing cooperation with potential users of the new solution. Thus, the application is designed and tested with users in every 
step of the iterative design process in order to enable a highly functional and usable outcome of the system.

In line with the ISO 13407 standard (Human-Centred Design Processes for Interactive Systems) the key aspects in our design process have been: 1) appropriate allocation of function between the user and the system, 2) active involvement of users, 3) iteration of design solutions, and 4) multi-disciplinary design team.

There is a range of different methods and techniques that can be used to achieve the goals of human-centred software development. The tools that have been applied within industry are e.g., planning, ISO standards, expert-based evaluation and inspection, early prototyping, usability performance evaluation, and subjective assessment. These techniques were, however, not seen suitable as such for the Lumetila design. As in every software solution, also in the Lumetila solution the user interface is the most critical part: it is the part of the system, which gives feedback and creates experiences to the user. Furthermore, in the Lumetila project the main aim is to create a totally new type of a user interface and to study its applicability in a game solution. Thus, to create our own method to user interface design when adapting the HCD approach was seen the most profitable way to come up with new, innovative ideas and to design an immersive, captivating and highly usable game (Figure 2).
In the Lumetila project the HCD process has been twofold:

1. State-of-the Art survey on experiences of computer game user interfaces as well as experiences on using the space or one's own body as a user interface in a game or an artwork.

2. Participatory evaluation; The potential end-users participate in the development process by carrying out scenario-based design together with the developers of the solution, evaluating the scenario-based storyboard, and testing the Lumetila prototype in Lumetila environment.

\section{Focus Areas}

During the state-of-the Art survey, the project concentrated on 5 focus areas that gave input to the design work. These focus areas, tightly connected to each other, were Game User Interface, Space User Interface, Body User Interface, Ergonomics and Safety, and Usability. In the area of Game User Interface, different games were used as a point of view when studying new ways for human-computer interactions. Concerning the Space User Interface, the project examined questions around user's possibilities to interaction, navigation and experience in many artistic installations and different free time applications known e.g., from amusement and science parks. In the area of Body User Interface, different interface solutions and ideas and their adequacy to the Lumetila prototype contexts were studied. Concerning Ergonomics and Safety, the project concentrated on risk analysis and safety level definition. The Usability area gave input to planning and evaluation of usability issues.

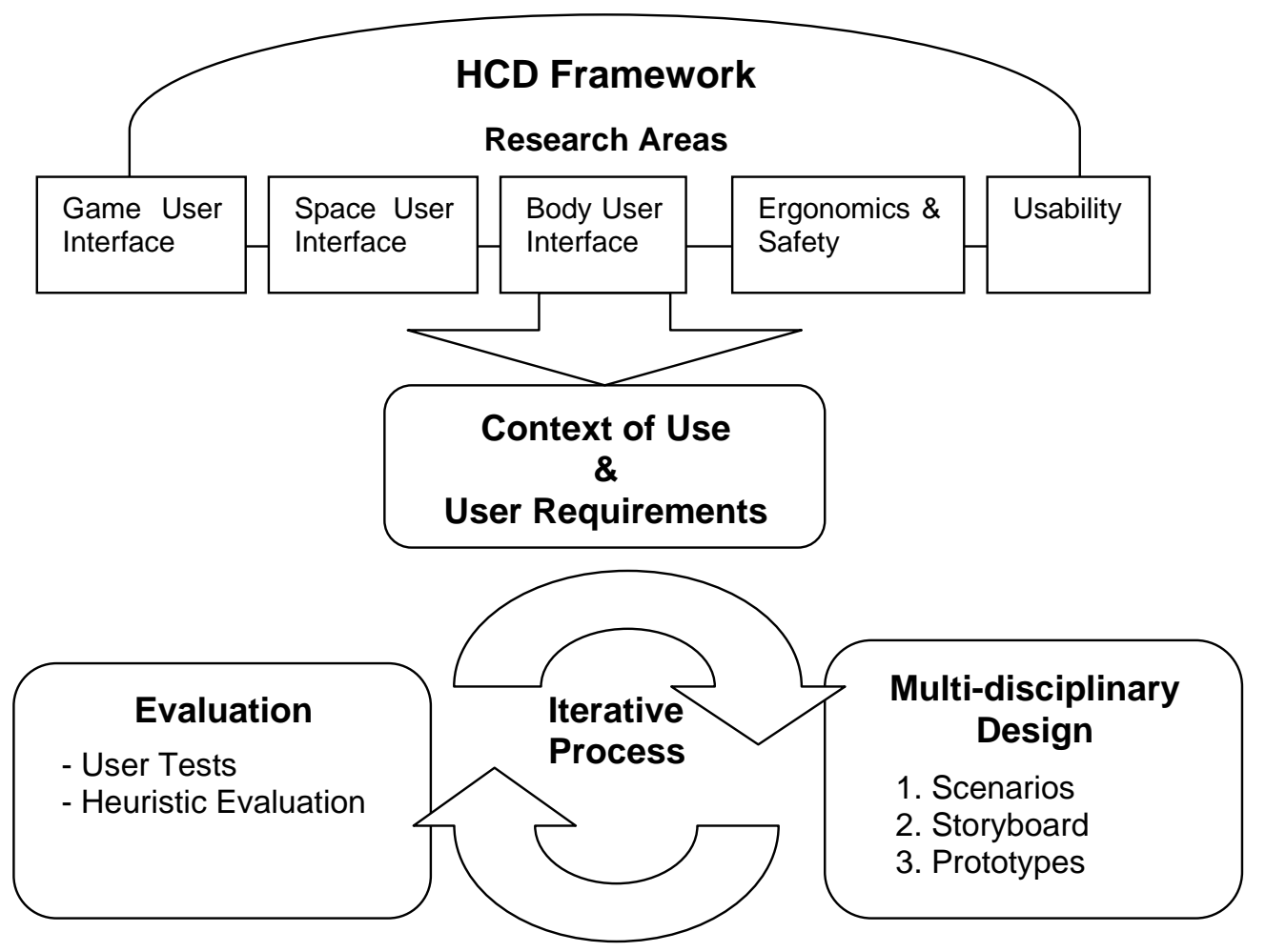

Figure 2. The Human-Centred Design Process and Approach of the Lumetila project. 


\section{Scenario-Based Design}

To generate design ideas, user requirements and usability goals, a basic method in Lumetila project has been Scenario-Based Design. The created scenarios were fictional stories of the Lumetila prototype, including the Virtual Space environment, the players and their desired activity, the events and the effects. They were also used as a tool for modelling user activity as well as planning and carrying out usability evaluation. The Lumetila project team created different genres for the Lumetila prototype in order to give input to the scenario work and the selection of the final scenario. The final scenario, which was based on the Group Balance genre, supports the idea of teamwork and interaction between the players. The players interact in the game by changing their position as a group on the floor, e.g., by running together to certain direction in the room.

\section{Storyboard}

The storyboard was created based on the scenario. It was evaluated in two sessions: The first evaluation was carried out as a pluralistic walk through with 20 school children who gave their opinions on the plot and the characters. Secondly, the storyboard was given to 19 families via Internet. After familiarising themselves with the storyboard they answered questions concerning the plot, the characters, and their interests in computer games in general. The storyboard was amended according the feedback from the user tests.

\section{LumePong as a test game}

The LumePong game (Figure 3) was created in order to test the functionality and relevance of the planned user interface of the Lumetila prototype. The technical environment to be used in the Lumetila prototype, especially the floor sensors and the player recognition, was tested with the users. The Virtual Space for the game was created at the Usability Laboratory of VTT Information Technology.

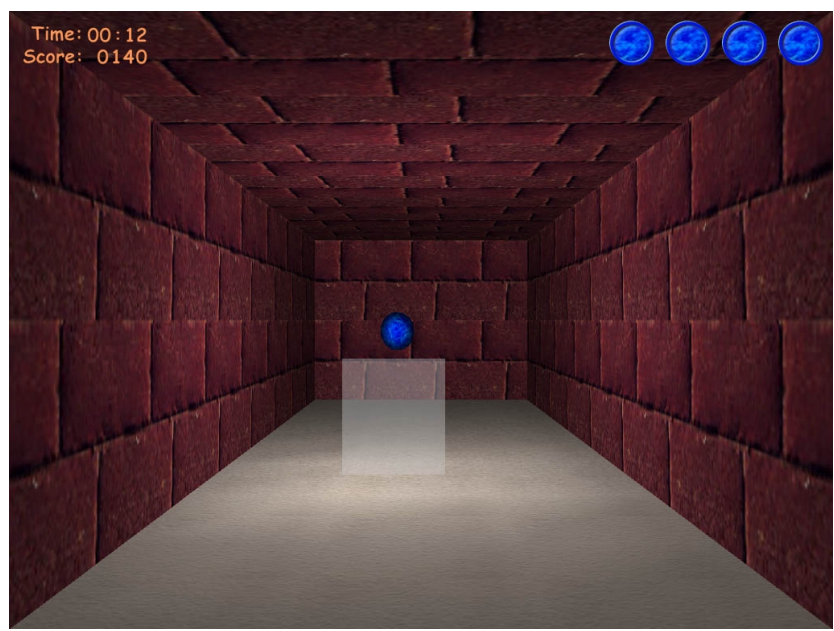

Figure 3. A screen shot of the LumePong game. The player controls the grey 'racquet' and tries to hit the ball by moving his body.
The LumePong game is based on the well-known Pong game. In our virtual space version there is a real time $3 \mathrm{D}$ graphics environment where the player controls a 'racquet' by moving around on the floor. The floor has pressure sensors for recognising the player's movements: when a player goes forward, the 'racquet' goes up, when he moves backwards the 'racquet' goes down, and so on.

\section{FINDINGS}

The HCD process and the chosen design methodology proved to be a practicable way to design the Virtual Space Game. Our approach focuses on a multi-disciplinary working method, which actively keeps in view the valuable opinions of the different members in the design group. It also efficiently allows the voices of the end-users to be heard in the very beginning and throughout the design process.

The project group found scenario-based design an appropriate and motivating method for the game design. The storyboard of the Lumetila prototype was created through many vibrant phases in the scenario building process.

To compare the differences and the similarities of the Lumetila idea to other virtual reality systems as well as to computer games' narrative, proved to be a good working method. Through this we could envisage the possible problems when designing Lumetila on the basis of earlier virtual reality systems or computer games' narrative and style. All the foundings were consoling.

As well as the floor sensor system, a real time 3D graphics engine and special effects devices are essential factors in creating the immersion and experience in the Virtual Space. The room for the Virtual Space is equipped with a 3D sound system and light effect devices. The fact that the players need not wear any virtual reality devices, e.g., data glasses or gloves, helps creating a total immersion, as well as starting the game and acting as a group.

\section{FUTURE WORK}

Based on the positive findings of the scenario work and the LumePong evaluation, VTT Information Technology will continue to develop the Lumetila prototype together with its partners. The Lumetila prototype will be a locationbased entertainment game suitable for people of all ages. It will not require any special skills to play it: using ones own body as a controller of the game will be the main factor in the game.

To carry on the iterative HCD process, also the Lumetila prototype will be evaluated in the Virtual Space room, described earlier, by usability experts (heuristic usability evaluation) and potential end-users. The evaluation results will be brought into the development work. One of the most interesting factor to be evaluated will be how well the Lumetila prototype will meet the original challenge of our design work: do we succeed in creating an interactive 
natural environment with a shared experience between the players.

\section{ACKNOWLEDGMENTS}

In the Lumetila project VTT Information Technology works in collaboration with Cube Ltd., Nokia Research Center, Tampereen Särkänniemi Ltd., and the University of Lapland. Tekes, the National Technology Agency, cofunds the project. We like to thank all the project participants.

\section{REFERENCES}

1. Bevan \& Macleod. (1994) Usability measurement in context. Behaviour \& Information Technology, 1994, VOL. 13, NOS.1 and 2, 132-145

2. Bias, R.G. (1994) The Pluralistic Usability Walkthrough Coordinated Emphathies. In Nielsen, J. \& Mack, R. (Eds.) Usability Inspection Methods. John Wiley and Sons, New York, NY. ISBN 0-471-01877-5

3. Daly-Jones O., Bevan N. \& Thomas C. (1997) Handbook of User-Centred Design. Telematics Applications Project IE 2016. European Usability Support Centres. Information Engineering Usability Support Centres.

4. ISO 13407. (1999) Human-centred design processes for interactive systems. International Organization for Standardization.
5. Johnson, P.J. (1992) Human Computer Interaction: psychology, task analysis and software engineering. McGraw Hill, London.

6. Maguire M. (1997) RESPECT User Requirements Framework Handbook. European Usability Support Centres. Requirements Engineering and Specification in Telematics.

7. Nielsen, Jakob. (1993) Usability Engineering. New York: Academic Press.

8. Nielsen, J. \& Mack Robert, L. (1994) Usability Inspection Methods, John Wiley \& Sons Inc.

9. Norman, D. A. (1993) Things that make us smart. Reading, MA: Addison-Wesley.

10.Preece, J. (1994) Human-Computer Interaction. Addison-Wesley.

11. Rubin, J. (1994) Handbook of usability testing. How to plan, design, and conduct effective tests. John Wiley \& sons Inc.

12.Räty, Veli-Pekka. (2000) Virtual Space - User Interfaces of the Future. VTT Information Technology. Available from http://www.vtt.fi/tte/projects/lumetila/ 